\title{
A realidade aumentada como recurso auxiliar para a aprendizagem significativa de
} geometria molecular

\author{
Augmented reality as auxiliary resource for significant learning of molecular geometry \\ Realidad aumentada como recurso auxiliar para el aprendizaje significativo de la geometría \\ molecular
}

Recebido: 03/10/2020 | Revisado: 10/10/2020 | Aceito: 04/08/2021 | Publicado: 09/08/2021

\author{
Nágila Menezes Rocha \\ ORCID: https://orcid.org/0000-0001-5007-8904 \\ Instituto Federal de Educação, Ciência e Tecnologia do Ceará, Brasil \\ E-mail: nagilamr7@gmail.com \\ Ana Karine Portela Vasconcelos \\ ORCID: https://orcid.org/0000-0003-1087-5006 \\ Instituto Federal de Educação, Ciência e Tecnologia do Ceará, Brasil
E-mail: karine @ifce.edu.br \\ Vicente Tomé do Nascimento Filho \\ ORCID: https://orcid.org/0000-0002-1693-1008 \\ Instituto Federal de Educação, Ciência e Tecnologia do Ceará, Brasil \\ E-mail: vicentenf@hotmail.com \\ Caroline de Góes Sampaio \\ ORCID: https://orcid.org/0000-0002-3642-234X \\ Instituto Federal de Educação, Ciência e Tecnologia do Ceará, Brasil \\ E-mail: carolinesampaio@ifce.edu.br \\ Maria Cleide da Silva Barroso \\ ORCID: https://orcid.org/0000-0001-5577-9523 \\ Instituto Federal de Educação, Ciência e Tecnologia do Ceará, Brasil \\ E-mail: carolinesampaio@ifce.edu.br
}

\begin{abstract}
Resumo
O conteúdo de Geometria Molecular é visto com dificuldade pela maioria dos estudantes. Muitas vezes essa dificuldade está ligada à maneira como o assunto é abordado em sala de aula. Sabe-se que as tecnologias digitais estão cada vez mais inseridas no cotidiano dos estudantes e que podem ser utilizadas para promover um abiente favorárel para a aprendizagem, auxiliando o professor em sala de aula. Com o intuito de facilitar o entendimento e promover uma aprendizagem significativa, o presente trabalho traz como sugestão a utilização da atividade proposta pelo aplicativo de realidade aumentada Mirage-Molecular Geometry, onde o aluno tem a oportunidade compreender a geometria de algumas moléculas através da observação de sua estrutura sob diversos ângulos. Além disso, a realidade aumentada promove uma educação de forma dinâmica, possibilitando o aluno participar ativamente da construção de seu conhecimento. Sendo assim, espera-se que a prática apresentada promova uma melhoria no processo de aprendizagem, tornando-o mais efetivo e atrativo.
\end{abstract}

Palavras-chave: Geometria molecular; Aprendizagem significativa; Realidade aumentada.

\begin{abstract}
The content of Molecular Geometry is viewed with difficulty by most students. This difficulty is often linked to the way the subject is approached in the classroom. It is known that digital technologies are increasingly inserted in the students' daily lives and that they can be used to promote a favorable environment for learning, helping the teacher in the classroom. In order to facilitate understanding and promote meaningful learning, the present work suggests the use of the activity proposed by the augmented reality application Mirage-Molecular Geometry, where the student has the opportunity to understand the geometry of some molecules through the observation of its structure from different angles. In addition, augmented reality promotes education dynamically, enabling students to actively participate in the construction of their knowledge. Thus, it is expected that the presented practice promotes an improvement in the learning process, making it more effective and attractive.
\end{abstract}

Keywords: Molecular geometry; Meaningful learning; Augmented reality.

\section{Resumen}

La mayoría de los estudiantes ven con dificultad el contenido de Geometría Molecular. Esta dificultad suele estar relacionada con la forma en que se aborda el tema en el aula. Se sabe que las tecnologías digitales se insertan cada vez 
más en la vida cotidiana de los estudiantes y que pueden utilizarse para promover un entorno favorable al aprendizaje, ayudando al docente en el aula. Con el fin de facilitar la comprensión y promover el aprendizaje significativo, el presente trabajo sugiere el uso de la actividad propuesta por la aplicación de realidad aumentada Mirage-Geometría Molecular, donde el alumno tiene la oportunidad de comprender la geometría de algunas moléculas a través de la observación de su estructura desde diferentes ángulos. Además, la realidad aumentada promueve la educación de forma dinámica, permitiendo que los estudiantes participen activamente en la construcción de sus conocimientos. Así, se espera que la práctica presentada promueva una mejora en el proceso de aprendizaje, haciéndolo más efectivo y atractivo.

Palabras clave: Geometría molecular; Aprendizaje significativo; Realidad aumentada.

\section{Introdução}

Uma das grandes dificuldades enfrentadas pelos professores de Química é a maneira de abordar conteúdos que exijam um certo nível de abstração dos alunos. O conteúdo de Geometria Molecular, que é abordado no primeiro ano do Ensino Médio, está inserido nesse grupo e necessita de uma atenção especial para que ele seja explorado de forma que favoreça a aprendizagem significativa, facilitando seu entendimento e distanciando-se da mecanização ainda bastante presente no cotidiano escolar.

De acordo com Moreira (2012) a aprendizagem significativa é aquela em que novas ideias interagem com conhecimentos já existentes na estrutura cognitiva do indivíduo, esses conhecimentos são considerados âncoras para que os novos conhecimentos adquiram significados.

A aprendizagem significativa de Ausbel é uma teoria que envolve o processo de construção do conhecimento, podendo ser classificada como cognitivista e construtivista (Masini,2011).

De acordo com Trindade (2011 apud Ausubel et al., 1980), a Aprendizagem Significativa "consiste na aquisição duradoura e memorização de uma rede complexa de ideias entrelaçadas que caracterizam uma estrutura organizada de conhecimento que os alunos devem incorporar em suas estruturas cognitivas".

Tomando como exemplo o estudo da Química, especificamente a geometria molecular, alguns conceitos podem ser considerados âncoras como a estrutura de Lewis de uma molécula e a Teoria da Repulsão dos Pares de Elétrons de Valência, pois permitem uma maior compreensão do conteúdo.

De acordo com Brown (2005) a estrutura de Lewis é uma representação dos elétrons de valência dos átomos que permitem seguir o rastro deles durante uma ligação química, representando também essas ligações. Esses elétrons são representados por um ponto para cada um ao redor da representação do elemento químico.

O segundo conhecimento âncora para mencionado é Teoria da Repulsão dos Pares de Elétrons de Valência (TRPEV) que é classificada por Brown (2005) como determinante para a geometria da molécula levando em consideração os elétrons ligantes e também não-ligantes.

Um par ligante de elétrons, portanto, define uma região no espaço, na qual é mais provável que os elétrons sejam encontrados. Chamaremos tais regiões de domínio de elétron. Igualmente, um par não-ligante (ou par solitário) de elétrons define um domínio de elétron localizado em certo átomo. (Brown, 2012,p.292)

Desse modo, visto que os domínios dos elétrons são carregados negativamente, eles têm a tendência de se repelirem, buscando a melhor posição para que o efeito da repulsão seja minimizado. Essa ideia é a base do modelo da REPV. Sendo assim, as diferentes formas espaciais dos íons ou moléculas dependem dos domínios de elétrons que rodeiam o átomo central.

(Brown, 2012)

Já a aprendizagem mecânica, ainda de acordo com o autor citado, se classifica como um processo praticamente sem significado, de natureza memorística, utilizado apenas para o processo avaliativo. Uma possibilidade para amenizar esse problema é a utilização da Realidade Aumentada (RA), um ambiente virtual em que o usuário pode fazer uma interação 
trazendo para o ambiente real objetos virtuais, incrementando e aumentando a visão do mundo real, favorecendo assim a aprendizagem significativa.

De acordo com Tori e Hussel (2018) a realidade aumentada é uma interface multimodal que leva o ambiente virtual ao espaço físico do usuário, utilizando dispositivos simples de interação e permitindo que essa seja feita de maneira natural, sem necessidade de treinamentos. Podendo ser usada em plataformas mais sofisticadas ou populares.

Além disso a Realidade Aumentada pode ser explorada de diversas maneiras, respeitando o estilo de aprendizagem de cada indivíduo. Ela propõe uma educação de forma dinâmica, e criativa, colocando o estudante como centro do processo de aprendizagem, fazendo com que ele seja ativo e construtor do seu próprio conhecimento (Rodrigues,2011).

Tal consideração leva a propor uma metodologia de trabalho em que, as atividades induzam os alunos a se questionarem sobre as influências das estruturas de Lewis e da Teoria da Repulsão dos Pares de Elétrons de Valência na geometria das moléculas, visando analisar a contribuição dessa tecnologia para a construção de uma Aprendizagem Significativa do conteúdo.

Além disso, há uma preocupação com a melhoria do processo de ensino-aprendizagem de Química. Sendo assim, propõe-se uma aplicação interativa de Realidade Aumentada como apoio didático no ensino de Química Geral, para os alunos de Ensino Médio. A atividade proposta traz como sugestão a utilização do aplicativo Mirage-Molecular Geometry.

Firmino et al. (2019) destaca que a utilização de aplicativos para dispositivos móveis pode ser uma aliada para o ensino de Química, pois possuem potencial para serem utilizados para auxiliar o professor nas aulas. Além disso, os recursos tecnológicos estão cada vez mais inseridos no cotidiano dos alunos.

De acordo com Silva, Sales e Silva (2019) a inserção de ferramentas digitais às aulas promove uma interação com o conhecimento, promovendo um ambiente propício para a aprendizagem. Além disso, a utilização de tecnologias que não exijam o uso de internet e computadores em sala de aula pode ser uma grande aliada para que escolas que não possuem uma infraestrutura inclusiva possa ter acesso a aula mais dinâmicas.

\section{Metodologia}

O estudo aqui realizado possui um breve caráter bibliográfico de natureza qualitativa, pois de acordo com Pereira et al 2018 a pesquisa qualitativa tem como foco principal o produto, os dados coletados são descritivos e a análise de dados tende a seguir um processo indutivo. Os autores mencionados no embasamento teórico foram selecionados com o intuito de oferecer uma melhor compreensão dos conceitos de aprendizagem significativa, utilizando com referência Moreira e Ausubel e apresentar o recurso da realidade aumentada e sua utilização na educação conforme descrito por Tori e Hussel.

Além disso, a presente pesquisa visa realizar uma interlocução entre os conceitos de aprendizagem significativa e da realidade aumentada para que o conteúdo de geometria molecular seja melhor compreendido pelos estudantes. A partir disso, propõe-se a utilização do aplicativo de Realidade Aumentada Mirage. O passo-a-passo de sua utilização será descrito adiante, tendo como caráter apenas sugestivo, ficando a sua adaptação à critério do usuário.

\section{Resultados e Discussão}

\subsection{Plataforma Sugerida}

A plataforma indicada, conforme já mencionado, é o aplicativo Mirage-Geometry Molecular. Ele é desenvolvido para estimular o compartilhamento de informações e debate em sala de aula, além de propor uma interação atrativa com o conteúdo, sendo o professor um auxiliador do processo. Será solicitado aos estudantes participantes para que na aula seguinte levem o um 
smartphone ou tablet à escola com o aplicativo Mirage-Molecular Geometry. Ele está disponível para as plataformas Android e iOS. Uma vez baixado, não necessitará de acesso à internet.

O aplicativo é acompanhado por 12 cartões que devem ser impressos, onde 6 cartões permitem colocar o problema para resolver e 6 outros cartões trazem elementos para resolução. Esses cartões trazem consigo um código QR que pode ser lido com o auxílio da câmera do aparelho. Os cartões de 1 a 6 são as moléculas em questão e os cartões de 7 a 12 trazem as estruturas de Lewis que ajudam a explicar a geometria das moléculas em questão, pois mostram se a molécula possui ou não pares de elétrons livres que influenciam diretamente no seu arranjo estrutural conforme indica a Tabela 1 a seguir.

Tabela 1 - Relação das moléculas que compõem a atividade.

\begin{tabular}{ccc}
\hline $\begin{array}{c}\text { Cartões } \\
\text { Problemas }\end{array}$ & Moléculas & $\begin{array}{c}\text { Cartões } \\
\text { de resposta }\end{array}$ \\
\hline Mapa 1 & Paracetamol & Mapa 12 \\
Mapa 2 & Metano & Mapa 7 \\
Mapa 3 & Amônia & Mapa 8 \\
Mapa 4 & Água & Mapa 9 \\
Mapa 5 & Dióxido de Carbono & Mapa 10 \\
Mapa6 & Metananmina & Mapa 11 \\
\hline
\end{tabular}

Fonte: Autores.

A Figura 1 mostra o exemplo do cartão-problema 4, que é a molécula de água, com seu respectivo cartão- resposta de número 9, contendo a estrutura de Lewis.

Figura 1 - Cartões que representam a molécula de água.

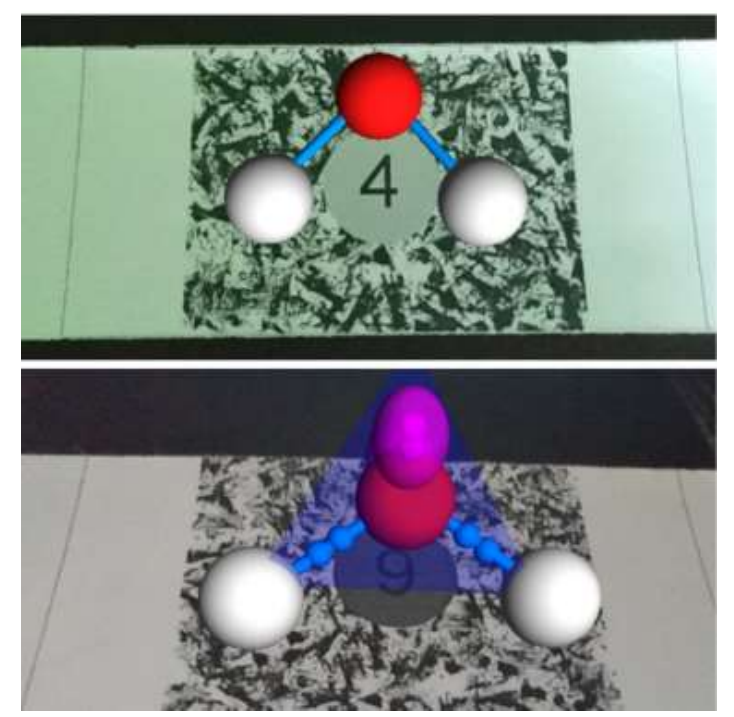

Fonte: Autores.

A partir da figura disposta acima pode-se perceber que no cartão-problema temos uma molécula com a geometria molecular do tipo angular, porém apenas no cartão-resposta fica claro que esse arranjo molecular só é possível pela presença dos pares de elétrons não ligantes, representados por meio de uma "nuvem eletrônica". 


\subsection{Atividade proposta}

O site Mirage dispõe de uma sugestão de atividade pedagógica para o professor que pode ser baixada através do site ( $h t t p: / / m i r a g e . t i c e d u . f r / ? p=2324$ ) a ser conduzida da seguinte forma:

1) Dividir os alunos em grupos de 2 a 4 podendo ser utilizado um tablet ou smartphone. Nesta etapa é contemplada um fator determinante para aprendizagem significativa, que é o compartilhamento de informações.

2) Aplicação de um vídeo explicativo sobre a estrutura de Lewis e revisão sobre alguns conceitos que estão nela inseridos. Aqui é possível observar a valorização dos conhecimentos prévios.

3) A partir desse momento inicia-se a avaliação diagnóstica. A molécula 1 (Figura 5) deverá utilizada como desencandeadora, reunindo no quadro as explicações dadas pelos alunos da geometria da molécula do paracetamol.

Figura 2 - Molécula desencandeadora da atividade.

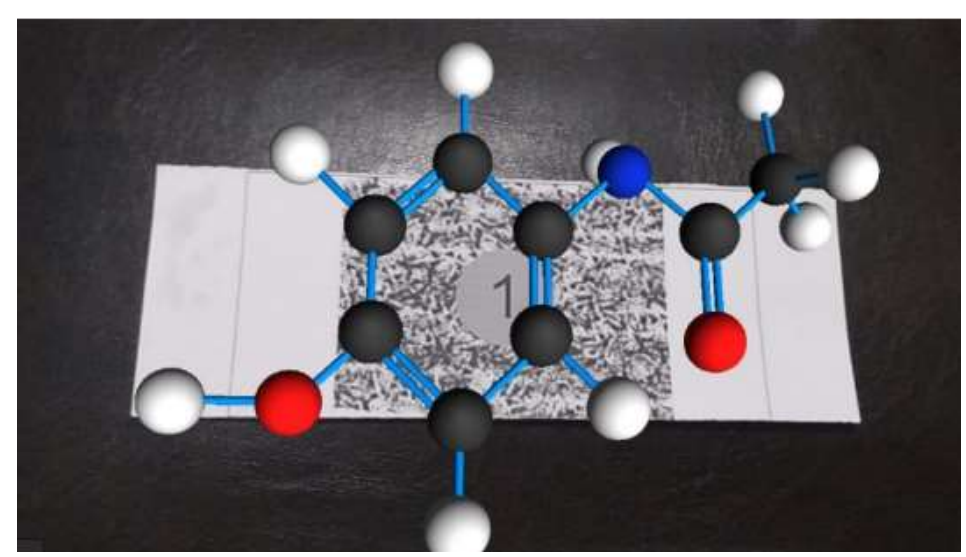

Fonte: Autores.

A figura acima nos permite observar que trata-se de uma estrutura de uma molécula que reúne diferentes tipos de geometria molecular, isso pode ser considerado um fator motivador para que os alunos tentem identificar e explicar essas geometrias através da observação sob diversos ângulos proporcionada pelo recurso da realidade aumentada. A seguir serão descritas as próximas etapas da atividade e serão realizados os comentários pertinentes a eles.

4) Cada grupo receberá uma carta entre os números de 2 a 6 (cada carta representa uma molécula diferente).

5) Será solicitado aos estudantes que escrevam algo sobre a geometria da molécula 1 e que explique a geometria da molécula que o grupo recebeu utilizando a estrutura de Lewis, os pares de elétrons e os pares de elétrons isolados.

6) Após os alunos darem suas próprias respostas sobre a geometria dessas moléculas serão distribuídos os cartões respostas que confirmarão ou corrigirão as respostas dadas pelos alunos. Este ponto põe em evidência mais uma vez a importância dos conhecimentos prévios para a determinação da geometria da molécula.

7) Em seguida será concedido um tempo para que os alunos organizem a apresentação da sua molécula para os demais alunos e professor, completando o quadro na lousa.

8) Para finalizar, será feito uma relação das moléculas simples que foram expostas e o cartão 12 para observar a geometria das diferentes partes da molécula paracetamol. Nesta etapa ocorre a incorporação efetiva e significativa dos novos conceitos apresentados, modificando finalmente a estrutura cognitiva. 


\section{Considerações Finais}

O objetivo central dessa pesquisa foi sugerir uma prática pedagógica que favorecesse ocorrência da aprendizagem significativa do conteúdo de geometria molecular através do auxílio da realidade aumentada. Esta interlocução é perfeitamente possível, pois como foi visto, alguns fatores essenciais para a aprendizagem significativa (como é o caso da motivação, participação ativa, conceitos prévios e trabalho em grupo) podem ser bastante explorados ao utilizar os diversos benefícios disponíveis por esse recurso.

O favorecimento da motivação pode ser explicado pelo uso do recurso tecnológico, que desperta a curiosidade e o interesse do aluno, visto que é um aparato que está cada vez mais presente no cotidiano deste. A participação ativa pode ser potencializada pelo recurso permitir a interação do indivíduo com o conteúdo, podendo neste caso observar as moléculas sob seus diversos ângulos, com ou sem par de elétrons livres.

Os conceitos prévios necessários para a melhor compreensão do conteúdo, como é o caso dos pares de elétrons de valência também podem ser facilmente visualizados através da utilização da realidade aumentada. Quanto ao trabalho em grupo, pode-se destacar que as interações e compartilhamentos de informações são peças fundamentais para a construção do conhecimento e podem ser exploradas pela atividade proposta pelo aplicativo ou de acordo com a preferência do docente. Ou seja, todas as características supracitadas vão ao encontro do objetivo comum que é a aprendizagem significativa.

A interação entre Aprendizagem Significativa e a Realidade Aumentada pode oferecer suportes teóricos e práticos indispensáveis para o trabalho docente, possibilitando uma melhor compreensão dos processos de ensino-aprendizagem, facilitando a aprendizagem significativa em diversos tópicos de Química, inclusive na Geometria Molecular. Espera-se também que o presente trabalho possa impulsionar futuras pesquisas que envolvam a aplicação prática da interlocução aqui apresentada, contribuindo para a melhoria do processo educativo.

\section{Referências}

Brown, T. L., Lemay Jr., H. E., \& Bursten, B. E. Química: A Ciência Central. Editora Pearson Education. (9a ed.).

Firmino, E. S., sampaio, C. G., Guerra, M. H. F. S., Nojosa, A. C. B., Saldanha, G. C. B., Vasconcelos, A. K. P. \& Barroso, M. C. S. (2019). Aplicativos móveis para uso no Ensino de Químico: uma breve análise. Research, Society and Development, 8(7), 1-14

Moreira, M. A. (2012). O que é a final aprendizagem significativa? Revista Qurriculum, La Laguna, 25, 29-56.

Masini, E. A. F. S. Aprendizagem Significativa: condições para ocorrência e lacunas que levam a comprometimentos. Aprendizagem Significativa em Revista/Meaningful Learning Review, 1(1), 16-24.

Mirage- Molecular Geometry. <http://mirage.ticedu.fr/?p=2324>.

Pereira, A. S., Shitsuka, D. M., \& Parreira, F. J., Shitsuka, R. Metodologia Da Pesquisa Científica. Editora UFSN.

Rodrigues, R. L. (2011) Realidade aumentada aplicada a educação: desenvolvimento de um protótipo utilizando rastreamento de mãos. Monografia, Universidade Estadual da Paraíba, Campina Grande, PB, Brasil.

Silva, D., Mourão, M., Sales, G., \& Silva, B. (2019). Metodologias ativas de aprendizagem: relato de experiência em uma oficina de formação continuada de professores de ciências. Revista De Ensino De Ciências E Matemática, 10(5), 206-223.

Trindade, J. O. Ensino e aprendizagem do conceito de ligações química por meio de mapas conceituais. 2011.230 f. Dissertação (Mestrado) - Curso de Mestrado Profissional em Química, Centro de Ciências Exatas e de Tecnologia, Universidade Federal de São Carlos.

Tori, R. \& Hounsell, M. S. (Eds.). (2018). Introdução a Realidade Virtual e Aumentada. Editora SBC 